\title{
Tunable Bandpass Transmission in Silicon Microring CROWS (0925133-PO)
}

\author{
SHAYAN MOOKHERJEA \\ Department of Electrical and Computer Engineering, University of California, San Diego, MC 0407 La \\ Jolla CA 92093-0407 USA \\ Email:smookher@ucsd.edu
}

Summary of a Project Outcomes report of research funded by the U.S. National Science Foundation under Project Number 0925133. A two-section optical filter is studied which can achieve tunable passband width and high extinction ratio.

\section{Introduction and Goals}

An optical filter comprising a ring resonator provides better stopband rejection at the cost of narrower passband as the pole moves closer to the unit circle. Increasing the contrast of a ring filter in this way results in very narrow passbands, which then make the filter thermally unstable, difficult to couple into, and inefficient in terms of spectral utilization.

This tradeoff can potentially be overcome using coupled-resonator optical waveguide (CROWs), proposed in 1999 by Yariv et al. [1]. CROWs are linear sequences of micro-resonators. The physical principle of light propagation is that of nearest-neighbor coupling between adjacent unit cells. In solid-state physics, this is known as "hopping conduction". A tight-binding model was used in early studies of optical CROWs [2] and was reformulated in a matrix eigenvalue problem to study the modes and resonance frequencies of the coupled-resonator system in more detail [3].

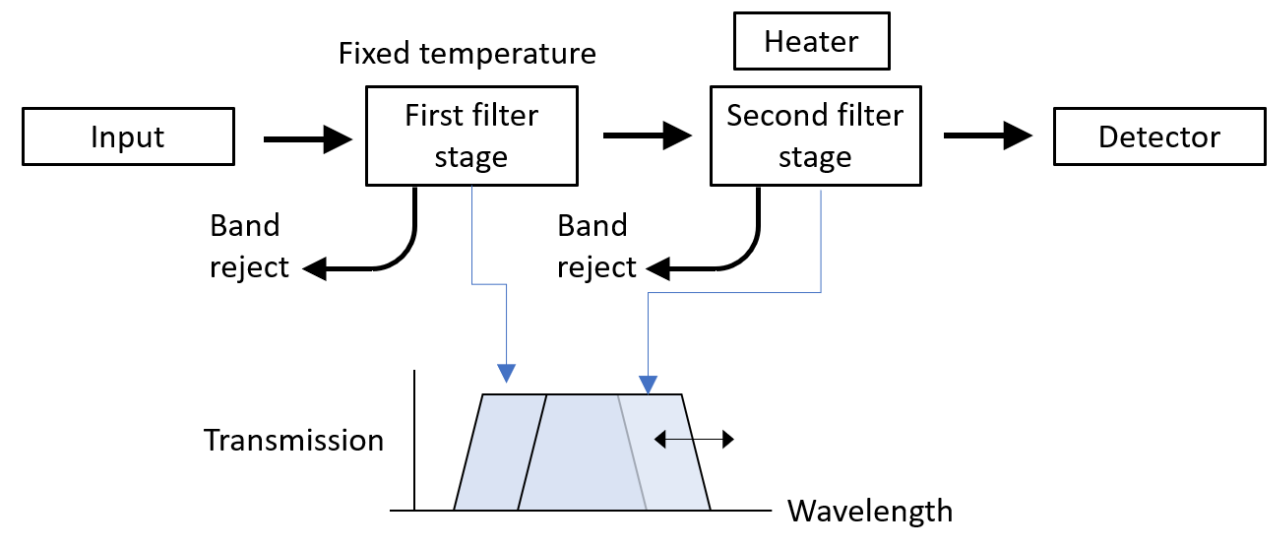

Figure 1 Concept of a two-section filter, where each filter stage consists of a CROW. 
Figure 1 shows the concept of a two section CROW-based filter. The passband of a CROW can be tuned by thermo-optic heating of the rings. If the passbands are initially aligned, the cascaded passband is narrowed as the second section is thermally shifted with respect to the other. If each section already achieves a high extinction ratio, then the overall extinction ratio is still acceptable when the passbands are shifted away from each other.

\section{Activities}

A more detailed and accurate computational method was developed for studying CROWs [4], [5]. Ab initio finite-difference time-domain simulation of long CROW structures is not practical, since disorder is manifest at the nanometer scale and the waveguides can be several millimeters in length. The nature of the onset of disorder was studied using these models, showing how the bandwidth gradually narrows and then abruptly collapses as the device length increases. These studies showed that, somewhat counter-intuitively, improved overall transmission and greater delay was achievable using a larger value of the inter-resonator coupling coefficient. Although the per-unit-cell phase (or time) delay is smaller, the waveguide is more robust to disorder, and longer CROW lengths can be realized [6].

CROWs of varying length were designed and fabricated. To experimentally measure these devices, new instrumentation was developed for high-resolution and rapid measurements of transmission and dispersion, demonstrating $1.4 \mathrm{pm}$ spectral resolution over $80 \mathrm{~nm}$ total bandwidth measured in a few seconds. We also used infrared imaging to study both single ring resonators and light propagation through CROW structures [7], [8].

a

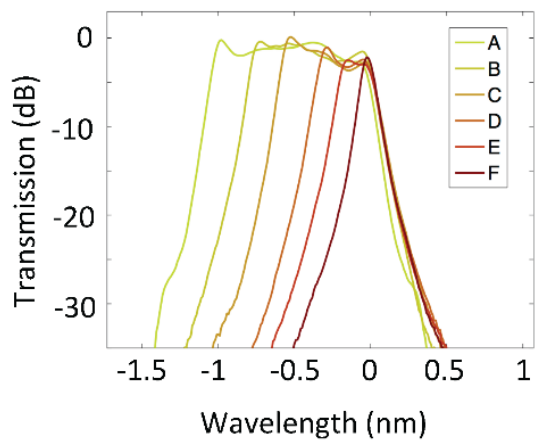

b

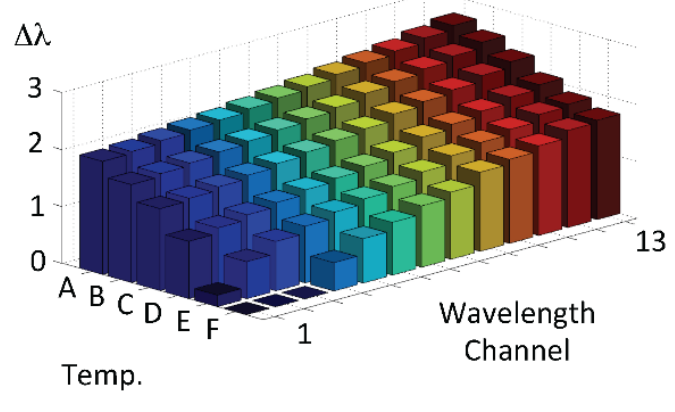

Figure 2 (a) The FWHM bandwidth of a CROW-based optical filter was reduced from $125 \mathrm{GHz}$ to $11.6 \mathrm{GHz}$. (b) Measurements of the CROW bandwidth reduction in discrete steps for multiple passbands across the $\mathrm{C}$ and $\mathrm{L}$ bands. 


\section{Progress and Outcomes}

The CROW devices were fabricated on SOI wafers. We divided a 10-ring CROW into two sections, each consisting of a $5^{\text {th }}$-order coupled-microring filter. The free-spectral range of the microrings was $7.3 \mathrm{~nm}$ near $1550 \mathrm{~nm}$ (bending radius $10 \mu \mathrm{m}$, directional coupler length $20 \mu \mathrm{m}$ ). Each section contributes $>50 \mathrm{~dB}$ contrast using only 5 microrings, with insertion loss (IL) $=$ $1.4 \mathrm{~dB}$. Cumulatively, the 10 rings had insertion loss (IL) $2.8 \mathrm{~dB}$. We used two separate chips to fully avoid thermal cross-talk, incurring an additional $9 \mathrm{~dB}$ transmission loss from the interconnections, which does not impact the measurement of either contrast or bandwidth.

The composite bandwidth was continually variable. Figure 3(a) shows the transmission through the CROW measured at discrete points along the tuning curve as it was reduced from a full-width at half-maximum (FWHM) bandwidth of $125 \mathrm{GHz}$ to $11.6 \mathrm{GHz}$.

The bandwidth was reduced by tuning the temperature of one section of five rings relative to the other (held constant at $23.2 \mathrm{C}$ ), with relative temperature increase, $\Delta \mathrm{T}$ at $0 \mathrm{C}$ (line labeled ' $\mathrm{A}$ '), 3C (line 'B'), 5.6C (line ' $\mathrm{C}$ '), 8.5C (line 'D'), 10.2 (line 'E') and 12C (line 'F').

Figure 2(b) shows the decrease in the edge-to-edge bandwidth occurs more rapidly for initially-narrow transmission channels, with a smaller value of the inter-resonator coupling coefficient. In all cases, increase of insertion loss, and eventual total transmission collapse, occurs after excessive detuning of one section of five rings relative to the other. The rate of bandwidth loss is more rapid for smaller coupling coefficients. Better performance from a CROW filter relies on strongly-coupled rings, with additional benefits arising from this, such as a widened bandwidth-to-FSR ratio, thermal stability, ease of coupling etc.

\section{Subsequent Extensions.}

A potential application of CROW filters is as part of an integrated photonic circuit following a nonlinear mixer device [9]. CROWs can also be used as tunable dispersion compensation devices [10].

Using the process of spontaneous four-wave mixing, the CROW structure can be used to generate entangled photon-pairs [11], [12]. The CROW naturally suppresses scattering noise in the pump in the out-of-band wavelengths, and can be designed to suppress Raman scattering from the pump, if the Raman peak lies in a stopband. However, a single silicon microresonator can also be used for the same purpose and can usually be controlled more easily [13] than a long CROW device.

Silicon is an electro-optically active material, and the propagation of CROW filters can also be tuned using voltage. This requires that $p-n$ junctions be 
incorporated across the waveguide cross-section [14]. The fastest tuning would be to incorporate a ferro-electric material, as has been shown with conventional Mach-Zehnder interferometers, but not yet with CROWs [15].

Microring resonators are sensitive to disorder. The results shown in Fig. 2 show that some trimming may be useful to further improve the flatness of the transmission and thereby, the cascaded transmission. We are studying potential methods for precisely trimming resonators separately from the heaters used to adjust the bandwidth. One method under investigation is based on field-induced local oxidation of $\mathrm{Si}$ to $\mathrm{SiO}_{2}$ via a chemical reaction near an electrically-biased conducting atomic-force microscope tip [16].

\section{Open-Access Reporting Initiative}

PRAISE: This open-access document is provided in support of our PRAISE

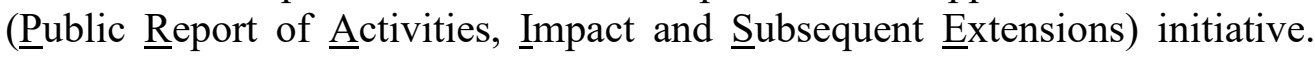
What is it? An open-access document shared with the public which describes the research outcomes of publicly-funded projects such as those funded by the U.S. NSF (National Science Foundation).

\section{References}

[1] A. Yariv, Y. Xu, R. K. Lee, and A. Scherer, "Coupled-resonator optical waveguide: a proposal and analysis," Opt. Lett., vol. 24, no. 11, pp. 711713, 1999.

[2] S. Mookherjea, D. S. Cohen, and A. Yariv, "Nonlinear dispersion in a coupled-resonator optical waveguide," Opt. Lett., vol. 27, no. 11, p. 933, Jun. 2002, doi: 10.1364/OL.27.000933.

[3] S. Mookherjea, "Spectral characteristics of coupled resonators," J. Opt. Soc. Am. B, vol. 23, no. 6, p. 1137, Jun. 2006, doi:

10.1364/JOSAB.23.001137.

[4] S. Mookherjea and M. A. Schneider, "Avoiding bandwidth collapse in long chains of coupled optical microresonators," Opt. Lett., vol. 36, no. 23, p. 4557, Dec. 2011, doi: 10.1364/OL.36.004557.

[5] M. L. Cooper and S. Mookherjea, "Modeling of Multiband Transmission in Long Silicon Coupled-Resonator Optical Waveguides," IEEE Photon. Technol. Lett., vol. 23, no. 13, pp. 872-874, Jul. 2011, doi: 10.1109/LPT.2011.2141657.

[6] M. L. Cooper et al., "235-ring Coupled-Resonator Optical Waveguides," in Conference on Lasers and Electro-Optics 2010, San Jose, California, 2010, p. CTuHH3. doi: 10.1364/CLEO.2010.CTuHH3.

[7] M. L. Cooper, G. Gupta, J. S. Park, M. A. Schneider, I. B. Divliansky, and S. Mookherjea, "Quantitative infrared imaging of silicon-on-insulator 
microring resonators," Opt. Lett., vol. 35, no. 5, p. 784, Mar. 2010, doi: 10.1364/OL.35.000784.

[8] S. Mookherjea and H. R. Grant, "High dynamic range microscope infrared imaging of silicon nanophotonic devices," Opt. Lett., vol. 37, no. 22, p. 4705, Nov. 2012, doi: 10.1364/OL.37.004705.

[9] J. R. Ong, R. Kumar, and S. Mookherjea, "Silicon microring-based wavelength converter with integrated pump and signal suppression," Opt. Lett., vol. 39, no. 15, p. 4439, Aug. 2014, doi: 10.1364/OL.39.004439.

[10] S. Mookherjea, "Using gain to tune the dispersion relation of coupledresonator optical waveguides," IEEE Photon. Technol. Lett., vol. 18, no. 5, pp. 715-717, Mar. 2006, doi: 10.1109/LPT.2006.871144.

[11] J. R. Ong and S. Mookherjea, "Quantum light generation on a silicon chip using waveguides and resonators," Opt. Express, vol. 21, no. 4, p. 5171, Feb. 2013, doi: 10.1364/OE.21.005171.

[12] R. Kumar, M. Savanier, J. R. Ong, and S. Mookherjea, "Entanglement measurement of a coupled silicon microring photon pair source," Opt. Express, vol. 23, no. 15, p. 19318, Jul. 2015, doi: 10.1364/OE.23.019318.

[13] M. Savanier, R. Kumar, and S. Mookherjea, "Optimizing photon-pair generation electronically using a $p-i-n$ diode incorporated in a silicon microring resonator," Appl. Phys. Lett., vol. 107, no. 13, p. 131101, Sep. 2015, doi: 10.1063/1.4932047.

[14] S. Mookherjea, J. R. Ong, X. Luo, and L. Guo-Qiang, "Electronic control of optical Anderson localization modes," Nature Nanotech, vol. 9, no. 5, pp. 365-371, May 2014, doi: 10.1038/nnano.2014.53.

[15] X. Wang, P. O. Weigel, J. Zhao, M. Ruesing, and S. Mookherjea, "Achieving beyond-100-GHz large-signal modulation bandwidth in hybrid silicon photonics Mach Zehnder modulators using thin film lithium niobate," APL Photonics, vol. 4, no. 9, p. 096101, Sep. 2019, doi: 10.1063/1.5115243.

[16] Y. Shen, I. B. Divliansky, D. N. Basov, and S. Mookherjea, "Perfect set-and-forget alignment of silicon photonic resonators and interferometers," in Optical Fiber Communication Conference/National Fiber Optic Engineers Conference 2011, Los Angeles, California, 2011, p. PDPC3. doi: 10.1364/OFC.2011.PDPC3. 